\title{
Chloroephedrine: contaminant of methamphetamine synthesis with cardiovascular activity
}

\author{
Kurt J. Varner ${ }^{\mathrm{a}, *}$, Nichole D. Hein ${ }^{\mathrm{b}}$, Brian A. Ogden ${ }^{\mathrm{a}}$, Jody R. Arsenault ${ }^{\mathrm{a}}$, \\ Karen M. Carter ${ }^{c}$, William H. Soine ${ }^{\mathrm{c}}$ \\ ${ }^{a}$ Department of Pharmacology, Louisiana State University Health Sciences Center, 1901 Perdido Street, New Orleans, LA 70112, USA \\ b Kalamazoo College, Kalamazoo, MI 49006, USA \\ ${ }^{\mathrm{c}}$ Department of Medicinal Chemistry, Virginia Commonwealth University, Richmond, VA 23298, USA
}

Received 1 November 2000; received in revised form 8 February 2001; accepted 8 February 2001

\begin{abstract}
Chloroephedrine is an intermediate and possible contaminant formed when methamphetamine is manufactured using ephedrine or pseudoephedrine as precursors. The purpose of this study was to determine whether this contaminant has biological activity and might contribute to methamphetamine-induced cardiovascular toxicity. In conscious rats, the $(-)$ and $(+)$ isomers of chloroephedrine ( 0.1 and $1.0 \mathrm{mg} / \mathrm{kg}$, i.v.) dose-dependently increased mean arterial pressure (MAP) and heart rate (HR). The potency of the pressor effects of $(-)$ and $(+)$-chloroephedrine was between that of ephedrine and pseudoephedrine. The increases in HR elicited by the four stimulants were similar except that the tachycardia elicited by all doses of ephedrine and pseudoephedrine were preceded by a brief decrease in HR. The i.v. administration of $10 \mathrm{mg} / \mathrm{kg}$ of $(+)$ or $(-)$-chloroephedrine produced biphasic (decrease followed by increase) the MAP and HR responses. Ephedrine and pseudoephedrine did not decrease MAP at any dose tested. The initial decrease in HR elicited by ( - )-chloroephedrine was significantly reduced and the hypotensive response abolished by atropine, indicating that these components of the MAP and HR responses resulted from vagal activation. The secondary pressor response elicited by ( - )-chloroephedrine was significantly reduced and the tachycardia significantly increased by pretreatment with phentolamine $(3 \mathrm{mg} / \mathrm{kg}$, i.v.). The increase in HR was reversed by propranolol. These results indicate that $(-)$ and (+)-chloroephedrine have sympathomimetic properties similar to other known sympathomimetic stimulants. In addition, larger doses of chloroephedrine can activate the vagus nerve. The combination of $(+)$-methamphetamine and $(-)$-chloroephedrine did not markedly alter the magnitude of the MAP and HR responses of $(+)$-methamphetamine alone except at high doses of $(-)$-chloroephedrine $(10 \mathrm{mg} / \mathrm{kg})$. Contamination of illicit methamphetamine with chloroephedrine may have toxic consequences. (C) 2001 Elsevier Science Ireland Ltd. All rights reserved.
\end{abstract}

Keywords: Blood pressure; Ephedrine; Heart rate; Illicit synthesis; Pseudoephedrine; Rat

\section{Introduction}

The illicit use of methamphetamine has become a major problem in the US and currently ranks among the top 20 drugs in emergency room and autopsy reports. The concentration of methamphetamine in blood and tissue samples in fatalities associated with methamphetamine is highly variable, with levels ranging from less than $1 \mathrm{mg} / 1$ to over $14 \mathrm{mg} / 1$ (Carvey and

\footnotetext{
* Corresponding author. Tel.: + 1-504-5684742; fax: +1-5045682361.

E-mail address: kvarne@1suhsc.edu (K.J. Varner).
}

Reed, 1970; Kojima et al., 1983; Matoba et al., 1985; Moore et al., 1996). In addition, there does not appear to be a close correlation between the blood levels of methamphetamine or amphetamine and survival (Karch et al., 1999). Often, low levels of amphetamines are thought to be incidental findings, but very low concentrations of methamphetamine $(0.7 \mathrm{mg} / \mathrm{l})$ have been observed in patients dying of what is often described as 'classic stimulant toxicity' with agitation, hypertension, tachycardia and hyperthermia (Fukunaga et al., 1987; Moore et al., 1996). Prior to the early 1970s, pharmaceutical grade methamphetamine was readily available. Currently, clandestinely synthesized 
methamphetamine is used almost exclusively on the 'street'. Forensic chemists have documented synthetic intermediates and impurities in samples of clandestinely synthesized methamphetamine; however, there is very little information regarding the contribution that these synthetic impurities might make to the pharmacology of clandestinely synthesized methamphetamine.

Many methods have been used to synthesize methamphetamine, but the most common current method involves the conversion of $(-)$-ephedrine or (+)-pseudoephedrine to $S$ - (+)-methamphetamine. Representative synthetic methods are depicted in Fig. 1 (Allen and Kiser, 1987). The common products or intermediates in this synthesis are the beta-halogenated amines. Synthesis from $(-)$-ephedrine yields almost exclusively the $(+)$ isomer of chloroephedrine. In contrast, synthesis from $(+)$-pseudoephedrine yields both the $(-)$ and $(+)$ isomers in a 40:60 ratio (Lekskulchai et al., in press). These compounds have been shown to cyclize to cis- or trans-1, 2-dimethyl-3-phenylaziridines. These intermediates, including the aziridines, are present in clandestinely synthesized methamphetamine (Allen and Kiser, 1987; Cantrell et al., 1988; Noggle et al., 1986; Skinner, 1990), but no quantitative data is included in those reports. However, in one of the reports the chromatogram of a forensic sample showed that similar amounts of methamphetamine and chloroephedrine were present (Noggle et al., 1986). The lack of reports documenting the presence of chloroephedrine in cases of methamphetamine overdose or fatality may reflect the conversion of the intermediates to ephedrine or pseudoephedrine during extraction and derivatization for analysis by gas chromatography/ mass spectrometry (Lekskulchai et al., in press). Given the ability of $(+)$-methamphetamine to produce cardiovascular and cardiac toxicity, a critical question that arises is whether these intermediates have biological activity and contribute to the pharmacological or toxicological responses elicited by $(+)$-methamphetamine. Therefore, the purpose of this study was to characterize the cardiovascular responses elicited by betachloroamines in conscious rats. The $(+)$ - and $(-)$ chloroephedrine- $\mathrm{HCl}$ salts were chosen for initial investigation due to the chemical stability of the $\mathrm{HCl}$ salts and the instability of formulations containing aziridines (Fruton, 1950). We also examined the role of adrenergic and cholinergic systems in the cardiovascular responses elicited by these compounds. For comparison, the cardiovascular responses elicited by the precursor compounds $(-)$-ephedrine and $(+)$-pseudoephedrine were also characterized. Pseudoephedrine and ephedrine have long been known to have sympathomimetic activity in many species including man (Dimson, 1934; Fristrom et al., 1997; Kitagawa and Saito, 1975; Martin et al., 1970). Finally we examined the MAP and HR responses elicited by $(+)$-methamphetamine with those elicited by combinations of $(+)$ methamphetamine and $(-)$-chloroephedrine.<smiles>CN[C@@H](C)[C@H](O)c1ccccc1</smiles>

(-)-ephedrine or

(+)-pseudoephedrine

$$
\downarrow \begin{aligned}
& \mathrm{SOCl}_{2} \\
& \text { or } \mathrm{PCl}_{5}
\end{aligned}
$$

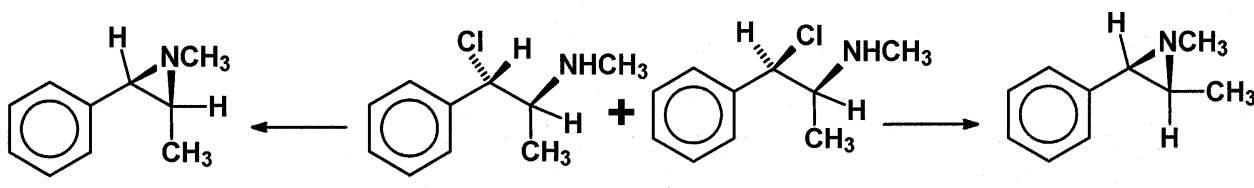

cis-1,2-dimethyl3-phenylaziridine (+)-chloroephedrine

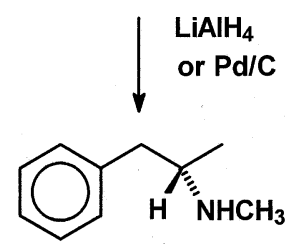

S-methamphetamine

Fig. 1. Flow diagram showing common clandestine methods used for the conversion of $(-)$-ephedrine or $(+)$-pseudoephedrine into (+)-methamphetamine via chloroephedrine intermediates. 


\section{Materials and methods}

\subsection{Subjects}

Experiments were performed on male Sprague-Dawley rats (275-350 g; Harlan, Indianapolis, IN). All procedures were in accordance with National Institutes of Health guidelines for the care and use of laboratory animals and were approved by the Institutional Animal Care and Use Committee at Louisiana State University Health Sciences Center. During the experiments the rats were housed individually in a room with a 12-hr light/ dark cycle. The rats had unlimited access to food and water. All testing was conducted on animals in their home cages.

\subsection{Surgical procedures}

To implant chronic cannulae, the rats were anesthetized using an i.p. injection of methohexital sodium (Brevital 40-45 mg/kg). Anesthesia was supplemented $(5-7 \mathrm{mg} / \mathrm{kg}$, i.v. or i.p.) in response to spontaneous movements or movements in response to tail or foot pinch. Body temperature was maintained at $37 \pm 1{ }^{\circ} \mathrm{C}$ using a water-filled heating pad and/or a heat lamp. Polyurethane catheters (Renethane, $0.014 \mathrm{~mm}$, I.D., 0.033 O.D.) were placed in the femoral artery and vein for the recording of arterial pressure (AP) and administration of drugs, respectively. The free ends of the cannulae were tunneled subcutaneously and exteriorized between the scapulae. Catheters were filled with heparinized saline $(200 \mathrm{U} / \mathrm{ml})$ to prevent clotting. Pulsatile AP and mean arterial pressure (MAP) were recorded using standard techniques (Abrahams et al., 1996). Heart rate (HR) was derived from the AP pulse using a Grass (Quincy, MA) 7P tachygraph. HR, MAP and pulsatile arterial pressure were displayed on a Grass (Quincy, MA) polygraph.

\subsection{Experimental procedures}

\subsubsection{Dose-response relationships for $M A P$ and $H R$ and $(+)$-chloroephedrine, $(-)$-chloroephedrine, $(-)$-ephedrine and (+)-pseudoephedrine}

Two days after implanting the cannulae the rats were divided into four groups containing five-six animals. After connecting the venous and arterial lines to the recording equipment, the animals were allowed $30 \mathrm{~min}$ to acclimate to the testing conditions and to achieve stable baseline cardiovascular recordings. Bolus doses of $(+)$-chloroephedrine $(0.1,1$ and $10 \mathrm{mg} / \mathrm{kg}),(-)$ chloroephedrine $(0.1,1.0$ and $10 \mathrm{mg} / \mathrm{kg}),(-)$ ephedrine $(0.1, \quad 0.5, \quad 1.0$ and $5 \mathrm{mg} / \mathrm{kg})$, or (+)-pseudoephedrine $(0.5,1.0,10$ and $50 \mathrm{mg} / \mathrm{kg})$ were administered i.v. over $5-10 \mathrm{~s}$ followed by a $10 \mathrm{~s}$ saline flush $(0.1 \mathrm{ml})$ and the changes in MAP and HR recorded. During dose-response testing each group of rats received only one of the stimulants. The 0.1 and 10 $\mathrm{mg} / \mathrm{kg}$ doses of $(-)$ and $(+)$-chloroephedrine were administered on the first day. In this and all subsequent dose-response experiments, the minimum inter-dose interval was $5 \mathrm{~h}$. The $1 \mathrm{mg} / \mathrm{kg}$ doses of $(-)$ and $(+)$-chloroephedrine were administered on the morning of the 2nd day. Likewise, the 0.1 and $10 \mathrm{mg} / \mathrm{kg}$ doses of $(-)$-ephedrine were administered on the morning and afternoon of the 1 st day, while the 0.5 and $1 \mathrm{mg} / \mathrm{kg}$ doses were given on the 2nd day. The 0.5 and $10 \mathrm{mg} / \mathrm{kg}$ doses of $(+)$ - pseudoephedrine were administered on the $1 \mathrm{st}$ day, while the 1 and $50 \mathrm{mg} / \mathrm{kg}$ doses were administered on the 2 nd day.

\subsubsection{Role of adrenergic and muscarinic mechanisms in mediating the MAP and $H R$ responses elicited by (-)-chloroephedrine}

Two days after completing the dose-response studies, ten rats from the ephedrine and pseudoephedrine groups were randomly selected and divided into two groups $(n=5)$. After connecting the arterial and venous lines and allowing the MAP and HR to achieve stable baseline values, one group was given an i.v. injection of the mixed alpha-adrenergic antagonist phentolamine (3 $\mathrm{mg} / \mathrm{kg}$ ). In preliminary studies, this dose of phentolamine completely blocked the pressor response elicited by the administration of phenylephrine $(9 \mu \mathrm{g} / \mathrm{kg})$. After the MAP and HR responses elicited by phentolamine had stabilized, ( - )- chloroephedrine $(10 \mathrm{mg} / \mathrm{kg}$, i.v.) was administered. After the responses to chloroephedrine had stabilized, the mixed beta-adrenergic antagonist propranolol ( $1 \mathrm{mg} / \mathrm{kg}$, i.v.) was administered. In the second group of rats the protocol was repeated except that the muscarinic antagonist atropine $(1 \mathrm{mg} / \mathrm{kg}$, i.v.), rather than phentolamine, was administered prior to injecting ( - )-chloroephedrine $(10 \mathrm{mg} / \mathrm{kg}$, i.v.). This dose of atropine completely blocked the MAP and HR responses elicited by acetylcholine (6 $\mu \mathrm{g} / \mathrm{kg}, \mathrm{i} . \mathrm{v}$.$) .$

\subsubsection{Cardiovascular responses elicited by the combined administration of methamphetamine and (-)-chloroephedrine}

In a separate group of rats $(n=6)$, cannulae were implanted in the femoral artery and vein as described above. Two days later, the cannulae were attached to the recording equipment and the baseline cardiovascular values allowed to stabilize. Each conscious rat was given a bolus i.v. injection of methamphetamine (2 $\mathrm{mg} / \mathrm{kg}$ ) delivered over $5-10 \mathrm{~s}$ followed by a saline flush $(0.1 \mathrm{ml})$ while the HR and MAP responses were recorded. Over the next 3 days HR and MAP were recorded as each of the rats received a single, daily bolus i.v. injection of methamphetamine $(2 \mathrm{mg} / \mathrm{kg})$ combined with either $0.1,1.0$ or $10 \mathrm{mg} / \mathrm{kg}$ of $(-)$ - 
Table 1

Baseline mean arterial pressure (MAP) and heart rate (HR) in the rats treated with either $(-)$ - ephedrine, $(+)$-pseudoephedrine, $(+)$ chloroephedrine or $(-)$-chloroephedrine

\begin{tabular}{lll}
\hline Treatment group & MAP $(\mathrm{mmHg})$ & HR $(\mathrm{bpm})$ \\
\hline (+)-chloroephedrine $(n=6)$ & $131 \pm 2$ & $385 \pm 10$ \\
(-)-chloroephedrine $(n=6)$ & $130 \pm 1$ & $399 \pm 7$ \\
(-)-ephedrine $(n=5)$ & $119 \pm 4^{\text {a,b }}$ & $364 \pm 24$ \\
$(+)$-pseudoephedrine $(n=5)$ & $123 \pm 2$ & $406 \pm 9$
\end{tabular}

\footnotetext{
a Indicates that the mean is significantly different from that in the (+)-chloroephedrine group.

${ }^{\mathrm{b}}$ Indicates that the mean is significantly different from that in the (-)-chloroephedrine group.
}

chloroephedrine. Each rat received all three combined doses of methamphetamine and ( - )-chloroephedrine.

\subsection{Data analysis}

The peak changes in HR and MAP elicited by administration of the four stimulants or combinations of methamphetamine and ( - )-chloroephedrine were calculated directly from the polygraph records. Baseline MAP and HR in the groups used for the dose-response studies were compared using one-way analysis of variance (ANOVA). Differences between individual means were compared using the Student-NewmanKuels method. The peak MAP and HR responses elicited by $(-)$-chloroephedrine before and after treatment with either atropine or phentolamine were compared using the Students $t$-tests. Significance was defined at $P<0.05$.

\subsection{Drugs used}

Drugs used were $(+)$-methamphetamine $\mathrm{HCl}$, atropine sulfate, phenylephrine $\mathrm{HCl}$, acetylcholine chloride, propranolol $\mathrm{HCl}$ and phentolamine methanesulfonate salt (Sigma Chemical, St. Louis, MO); methohexital sodium (Jones Pharma, St. Louis, MO) and ( - )-ephedrine and (+)-pseudoephedrine (Spectrum Quality Products, New Brunswick, NJ). The $(-)$ and $(+)$ isomers of chloroephedrine were synthesized as previously reported (Lekskulchai et al, in press) and were $\geq 98 \%$ a single diastereomer.

\section{Results}

\subsection{Dose-response relationships for $M A P$ and $H R$ and $(+)$-chloroephedrine and (-)-chloroephedrine, $(-)$ - ephedrine and $(+)$-pseudoephedrine}

Table 1 shows the baseline levels of MAP and HR in the four groups of rats used in the dose-response studies. Baseline MAP in the group that received ( - )ephedrine was significantly lower than the baseline MAP in the $(+)$ and $(-)$-chloroephedrine groups (Table 1). Baseline HR was similar in all four groups.

Fig. 2 compares the dose-response relationships for MAP and HR and the four stimulants tested. The
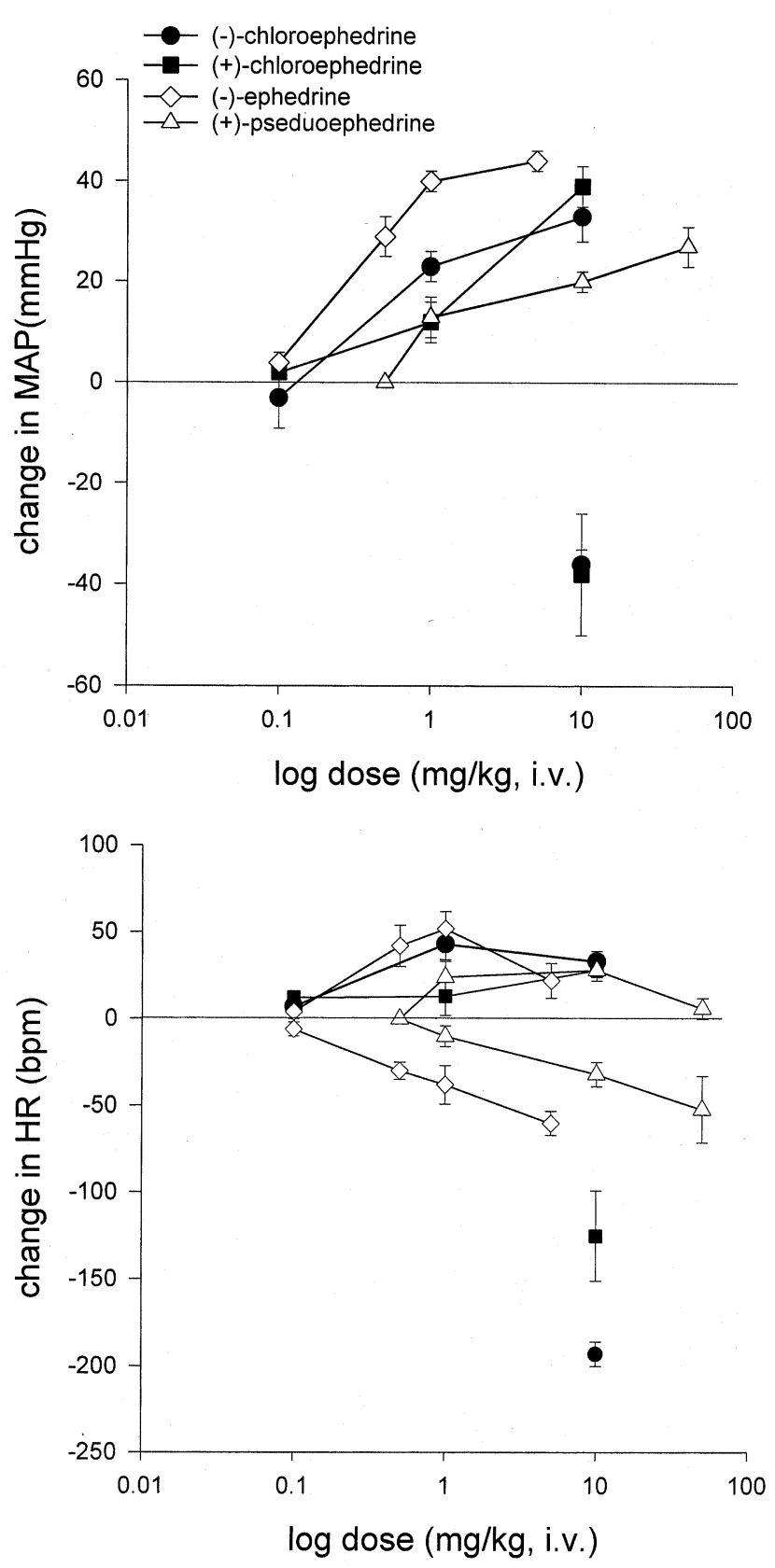

Fig. 2. Dose-response relationships for mean arterial pressure (MAP) and heart rate $(\mathrm{HR})$ and $(-)$-chloroephedrine $(n=6), \quad(+)$ chloroephedrine $(n=6),(-)$-ephedrine $(n=5)$ and $(+)$-pseudoephedrine $(n=5)$ in conscious rats. Top panel shows the magnitude of the peak changes in MAP elicited by i.v. administration of the four drugs. Bottom panel shows the magnitude of the peak changes in HR. When drug administration elicited bidirectional (decreases followed by increases) MAP and HR responses, both the peak decrease and peak increase are plotted. 
(+) chloroephedrine

(-) chloroephedrine

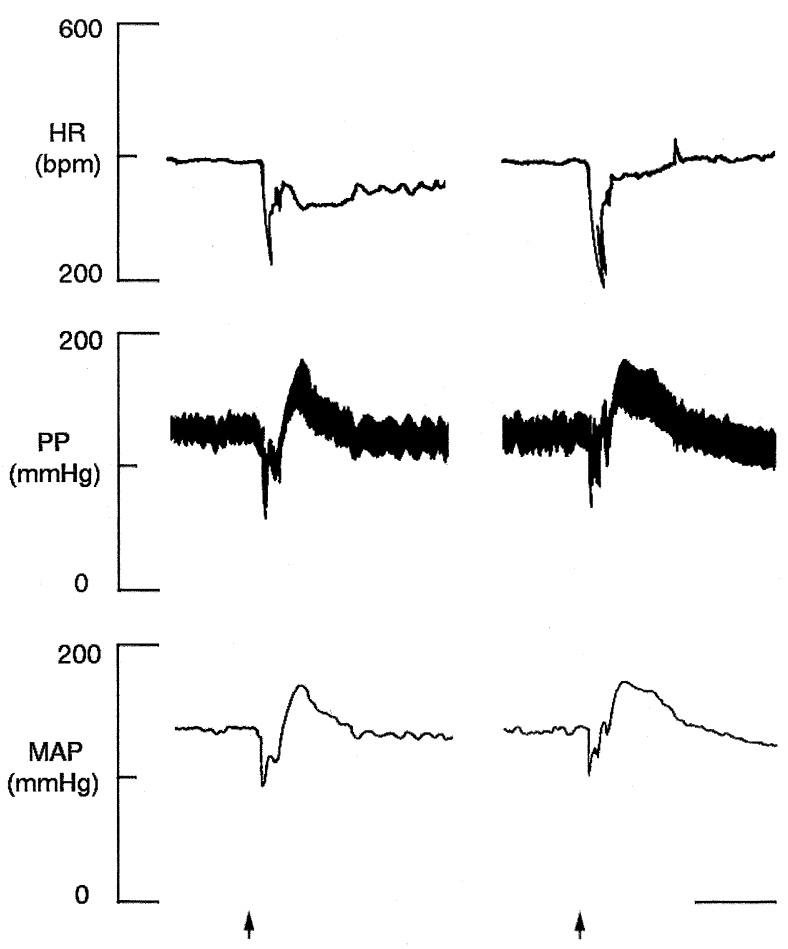

Fig. 3. Polygraph records showing typical HR, pulse pressure (PP) and MAP responses elicited by the i.v. administration of $10 \mathrm{mg} / \mathrm{kg}$ of $(+)$-chloroephedrine and $(-)$-chloroephedrine in conscious rats. Abbreviations are the same as in Fig. 2. Arrow denotes time of drug administration. Horizontal calibration is $1 \mathrm{~min}$.

MAP responses elicited by both isomers of chloroephedrine were very similar. The lower doses of both chloroephedrine isomers increased MAP; however, administration of the $10 \mathrm{mg} / \mathrm{kg}$ doses elicited biphasic MAP responses consisting of marked decreases in MAP followed by hypertension (Figs. 2 and 3). The pattern of HR responses elicited by the two isomers of chloroephedrine mirrored the MAP responses. The low doses of either isomer increased HR, while the 10 $\mathrm{mg} / \mathrm{kg}$ doses elicited biphasic HR responses consisting of large decreases in HR followed by increases (Figs. 2 and 3$)$. The bradycardia elicited by the $(+)$ isomer was smaller than that elicited by the $(-)$ isomer (Fig. 2). Typical polygraph records illustrating the biphasic MAP and HR responses elicited by the $10 \mathrm{mg} / \mathrm{kg}$ doses of $(-)$ and $(+)$ chloroephedrine are shown in Fig. 3.

In contrast to the $(-)$ and $(+)$ isomers of chloroephedrine, only increases in MAP were elicited by the doses of $(-)$-ephedrine and (+)-pseudoephedrine tested (Fig. 2). At all doses, the pressor responses elicited by $(-)$-ephedrine and $(+)$-pseudoephedrine were accompanied by a biphasic (decrease then increase) HR responses (Fig. 2). While the increases in HR elicited by all four of the stimulants were similar, the decreases in HR elicited by the $10 \mathrm{mg} / \mathrm{kg}$ doses of $(-)$ and $(+)$-chloroephedrine were larger than those elicited by the highest doses of $(-)$ ephedrine or $(+)$-pseudoephedrine.

\subsection{Role of adrenergic and muscarinic mechanisms in mediating the MAP and $H R$ responses elicited by (-)-chloroephedrine}

Fig. 4 shows representative polygraph records of the MAP and HR responses elicited by (-)chloroephedrine $(10 \mathrm{mg} / \mathrm{kg})$ during control and after pretreatment with phentolamine or atropine. These data are summarized in Fig. 5. Pretreatment with phentolamine $(n=5)$ did not alter the magnitude of the initial depressor or bradycardic components of the responses to ( - )-chloroephedrine (Figs. 4 and 5). However, in the presence of phentolamine the magnitude of the pressor response elicited by $(-)$-chloroephedrine was significantly decreased (Figs. 4 and 5). The attenuated pressor response was followed by a secondary decreased in MAP of $-17 \pm 7 \mathrm{mmHg}$ below prechloroephedrine levels (Fig. 4). Pretreatment with phentolamine significantly increased the tachycardic response to ( - )-chloroephedrine (Figs. 4 and 5). The peak of the increase in HR coincided with the secondary fall in MAP (Fig. 4). The increase in HR and secondary decrease in MAP elicited by $(-)$ chloroephedrine in the phentolamine-treated rats were reversed $(479 \pm 2$ to $305 \pm 24 \mathrm{bpm}$ and $68 \pm 1$ to $117 \pm$ $2 \mathrm{mmHg}$, respectively) by the administration of propranolol (1 $\mathrm{mg} / \mathrm{kg}$, i.v.). Pretreatment with atropine significantly reduced the magnitude of the bradycardic response and abolished the depressor response elicited by ( - )-chloroephedrine (Figs. 4 and 5). Atropine did not significantly change the magnitude of the tachycardic response elicited by $(-)$-chloroephedrine (Fig. 5).

\subsection{Cardiovascular responses elicited by the combined administration of $(+)$-methamphetamine and $(-)$-chloroephedrine}

In conscious rats the i.v. administration of methamphetamine $(2 \mathrm{mg} / \mathrm{kg})$ increased MAP and produced a biphasic (decrease then increase) HR response (Fig. 6). The magnitude of the pressor response elicited by 2 $\mathrm{mg} / \mathrm{kg}$ of $(+)$-methamphetamine was not altered by the addition of ( - )-chloroephedrine (Fig. 6). However, in three of six, rats when $1 \mathrm{mg} / \mathrm{kg}$ of (-)chloroephedrine was combined with methamphetamine the increases in MAP were preceded by depressor responses (Fig. 6). The addition of 0.1 and $1.0 \mathrm{mg} / \mathrm{kg}$ of $(-)$-chloroephedrine did not alter the magnitude of the HR rate responses elicited by $(+)$-methamphetamine. However, the combination of $(+)$-methamphetamine with $10 \mathrm{mg} / \mathrm{kg}$ of $(-)$-chloroephedrine elicited an in- 
crease in HR that was significantly greater than that elicited by either drug alone (Fig. 6). Moreover, the bradycardic responses elicited by the administration of $10 \mathrm{mg} / \mathrm{kg}$ of $(-)$-chloroephedrine or $(+)$-methamphetamine $(2 \mathrm{mg} / \mathrm{kg})$ alone were abolished when these drugs were given in combination (Fig. 6).

\section{Discussion}

The $(+)$ and $(-)$ isomers of chloroephedrine are contaminants that can be produced during the illicit manufacture of $(+)$-methamphetamine when $(+)$ pseudoephedrine or $(-)$-ephedrine are used as precursors. Chloroephedrine has been reported to be present in varying levels in clandestinely synthesized methamphetamine (Allen and Kiser, 1987; Cantrell et al., 1988; Noggle et al., 1986; Skinner, 1990) and has been observed in some forensic samples (Noggle et al., 1986). An anecdotal report suggests that chloroephedrine impurities can be present in methamphetamine manufactured by 'unscrupulous and/or unskilled' chemists and that these impurities 'ruin the finer aspects of the meth high' (Fester, 1999). The actual amounts of impurities in samples of clandestinely synthesized methamphetamine have not been reported. This lack of knowledge is most likely due to methodological difficulties encountered in extracting and quantifying the impurities (Lekskulchai et al., in press). Noggle and colleagues (1986) provided chromatographic evidence suggesting that some forensic samples contained equal amounts of methamphetamine and chloroephedrine. Based on our experience with the synthetic pathways used in the clandestine manufacture of methamphetamine, an impurity content of $0-40 \%$ or higher is possible, especially if an unskilled chemist manufactures the drug. Since some methamphetamine abusers administer doses as large as $1000 \mathrm{mg}$ (Konuma, 1994), it is possible that $400 \mathrm{mg}$ or greater of chloroephedrine may be consumed. Since rats may be less sensitive than humans to the cardiovascular actions of chloroephedrine, our dose-response and combination studies employed doses of chloroephedrine that were similar to- and exceeded potential human doses.

To our knowledge, the present data are the first to demonstrate that chloroephedrine can alter cardiovascular function/activity in conscious rats. Both isomers of chloroephedrine produce similar dose-dependent increases in MAP in conscious rats. The potency of the pressor actions of the $(+)$ and $(-)$ isomers was between that of $(-)$-ephedrine and $(+)$-pseudoephedrine. Our results in conscious rats confirm earlier reports that $(-)$-ephedrine is several times more potent than $(+)$-pseudoephedrine as a pressor agent control
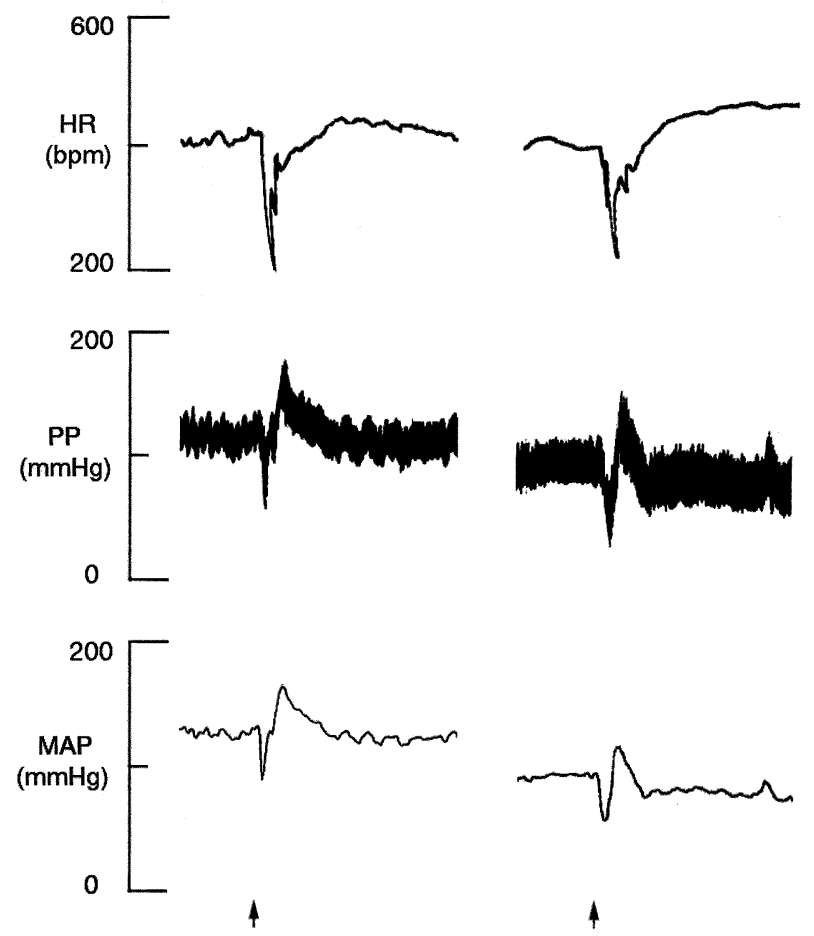

phentolamine
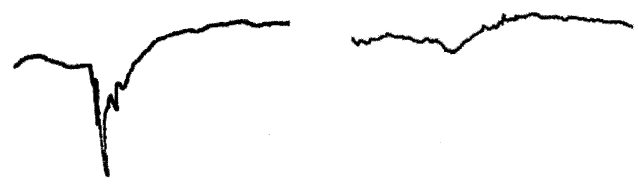

atropine

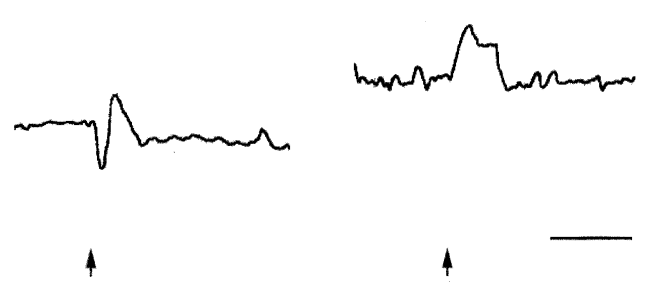

Fig. 4. Polygraph records showing the MAP, PP and HR responses elicited by the i.v. injection of $(-)$-chloroephedrine $(10 \mathrm{mg} / \mathrm{kg}$, i.v.) in untreated rats and rats pretreated with either phentolamine $(3 \mathrm{mg} / \mathrm{kg}$, i.v.) or atropine $(1 \mathrm{mg} / \mathrm{kg}$, i.v.). Abbreviations and format are the same as in Fig. 3. Horizontal calibration is $1 \mathrm{~min}$. 

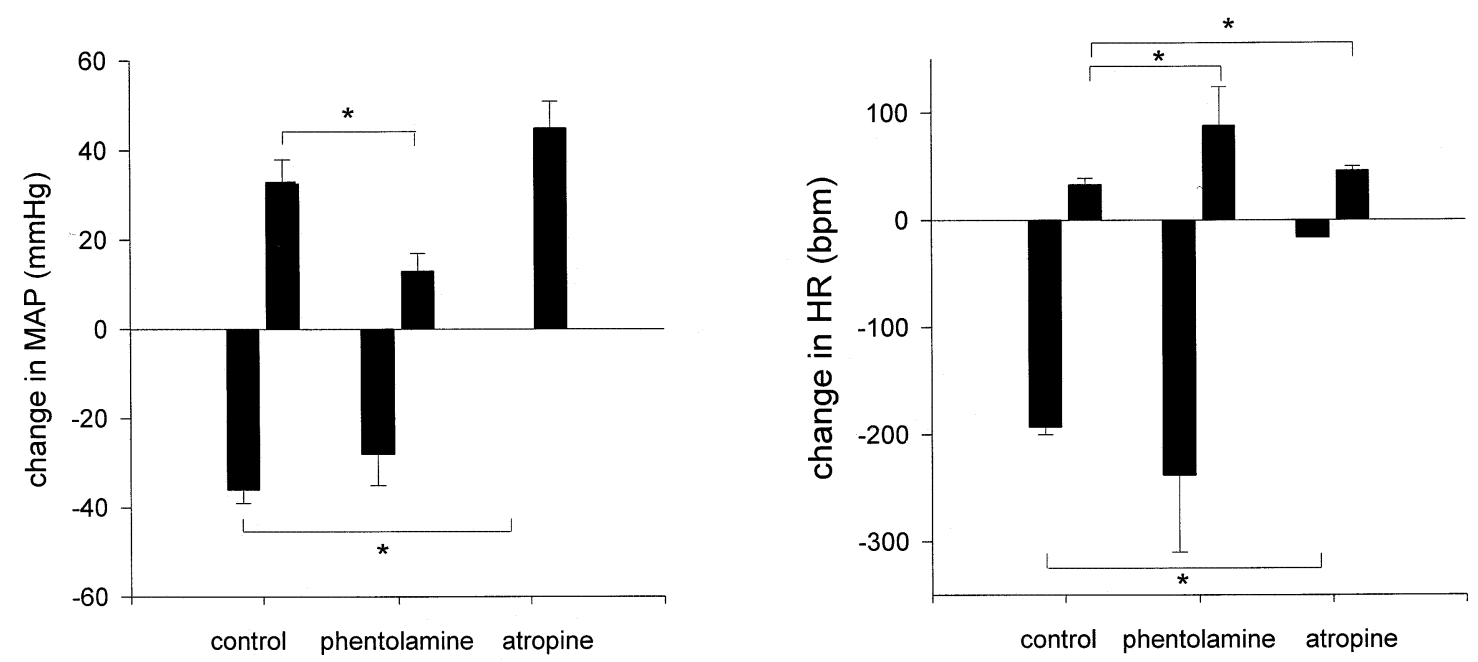

Fig. 5. Summary of the peak changes in MAP and HR elicited by the i.v. administration of ( - )-chloroephedrine ( $10 \mathrm{mg} / \mathrm{kg}$, i.v.) in untreated conscious rats (control) and in rats pretreated with either phentolamine $(3 \mathrm{mg} / \mathrm{kg}$, i.v.) or atropine $(1 \mathrm{mg} / \mathrm{kg}$, i.v.). $N=5 \mathrm{in}$ each group. Abbreviations are the same as in Fig. 2. * denotes significant difference $P<0.05$ between means.

(see Dimson, 1934; Drew et al., 1978). In all cases, the increases in MAP elicited by the isomers of chloroephedrine were accompanied by tachycardia. In contrast, ephedrine and pseudoephedrine produced biphasic HR responses consisting of a brief bradycardia that coincided with the pressor response, followed by tachycardia. Although not tested, it is likely that the bradycardia resulted from pressor-mediated baroreceptor reflex activation.

It is widely accepted that the sympathomimetic actions of both ( - )-ephedrine and (+)-pseudoephedrine involve a drug-mediated release of catecholamines from peripheral sympathetic nerves and a direct agonist action at peripheral adrenergic receptors (Pentel, 1984). To determine the role of peripheral alpha-adrenergic receptors in mediating the pressor and tachycardic responses elicited by chloroephedrine, we compared the MAP and HR responses elicited by $(-)$ chloroephedrine before and after complete alpha-adrenergic receptor blockade using phentolamine. Since the MAP and HR responses elicited by the $10 \mathrm{mg} / \mathrm{kg}$ dose of the $(-)$ and $(+)$ isomers were similar, only the $(-)$ isomer was tested in the antagonist studies. Pretreatment with phentolamine significantly attenuated the magnitude of the pressor response elicited by $10 \mathrm{mg} / \mathrm{kg}$ of (-)-chloroephedrine, indicating that the pressor response resulted from the activation of vascular adrenergic receptors. Whether chloroephedrine releases catecholamines from sympathetic nerve terminals and adrenal gland, or has direct alpha-adrenergic agonist effects remains to be determined. Pretreatment with phentolamine also significantly increased the magnitude of the tachycardic response elicited by (-)chloroephedrine. The increase in HR coincided with a secondary decrease in MAP, raising the possibility that the increased tachycardia reflected a decrease in baroreceptor reflex activity. The reflex-mediated increase in HR should have been enhanced by the blockade of inhibitory presynaptic alpha-2 receptors on cardiac sympathetic neurons. The increase in HR and the secondary hypotension elicited by $(-)$-chloroephedrine in phentolamine-treated rats were reversed by propranolol, indicating the involvement of cardiac and vascular beta-adrenergic receptors, respectively. Whether the beta-adrenergic stimulation arises from the sympathetic nerves, adrenal gland or a direct agonist action of chloroephedrine remains to be determined.

A striking difference between the cardiovascular responses elicited by larger doses of the isomers of chloroephedrine and those elicited by either $(-)$ ephedrine or $(+)$-pseudoephedrine were the large, concurrent decreases in MAP and HR that preceded the secondary pressor and tachycardic responses. The simultaneous decreases in HR and MAP elicited by these doses of chloroephedrine resemble those produced by activation of the Bezold-Jarisch reflex (Somers and Mark, 1996; Thoren, 1979). This vaso-vagal cardiac reflex is initiated by the chemical or mechanical stimulation of cardiac vagal afferents and subsequent activation of cardiac vagal efferents which slow HR, decrease cardiac output and lower AP (Thoren, 1979). The ability of atropine to significantly attenuate the initial decrease in HR and completely abolish the decrease in MAP elicited by $10 \mathrm{mg} / \mathrm{kg}$ of (-)-chloroephedrine, indicates that high doses of chloroephedrine produce vagal activation.

There are numerous reports linking the illicit use of ephedrine and ephedrine-containing compounds with severe and often fatal cardiovascular toxicity (Josefson, 1996; Pentel, 1984; Theoharides, 1997; Zahn, et al., 
1999). Our observation that the isomers of chloroephedrine produce a pattern of $\mathrm{HR}$ and MAP responses similar to those elicited by ephedrine raises the possibility that these compounds may also produce cardiovascular toxicity. The isomers of chloroephedrine can also produce vagal activation, which could result in syncope or increase the potential for certain types of cardiac arrhythmia. Since the chloroephedrines and the sympathomimetic stimulants elicited similar MAP and HR responses, the question arises as to whether contamination with the chloroephedrines has the potential to produce additive
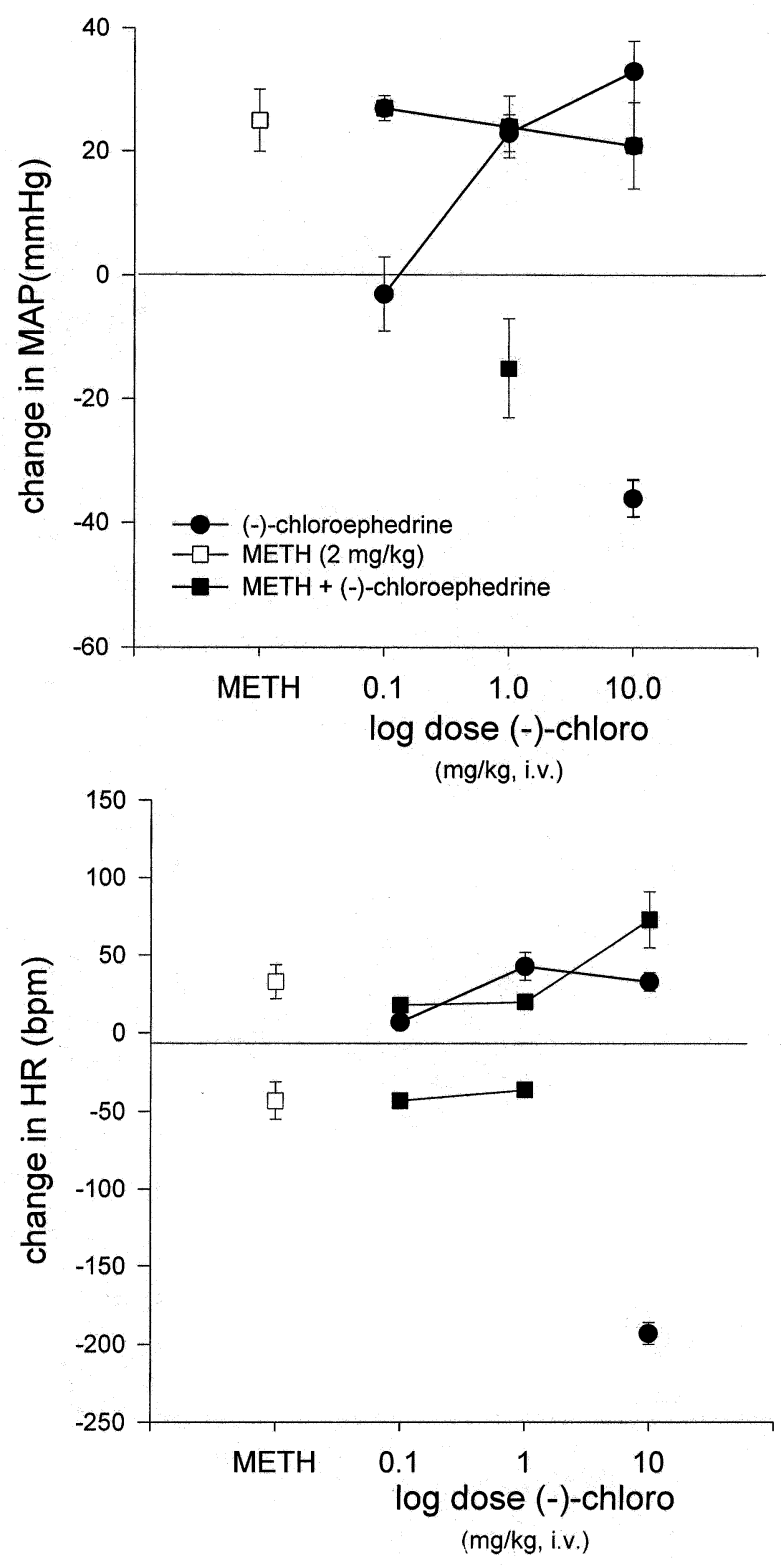

Fig. 6. Summary of the peak changes in MAP and HR elicited by the i.v. administration of methamphetamine $(2 \mathrm{mg} / \mathrm{kg}, n=6)$ or methamphetamine combined with 0.1 .1 .0 or $10 \mathrm{mg} / \mathrm{kg}$ of (-)chloroephedrine. Abbreviations are the same as in Fig. 2. or synergistic cardiovascular responses. In preliminary studies (see Fig. 6), the combination of $(+)$-methamphetamine and $10 \mathrm{mg} / \mathrm{kg}$ of $(-)$-chloroephedrine produced a tachycardic response that was significantly larger than that produced by methamphetamine or chloroephedrine alone. This was the only case were there was an additive effect of chloroephedrine and methamphetamine on the MAP or HR responses. While it appears that contamination with chloroephedrine does not markedly alter the magnitude of the MAP and HR responses elicited by methamphetamine, we cannot rule out the possibility that more complex interactions may be occurring. These complex interactions could affect other types of methamphetamine-related toxicity, such as cardiac toxicity, seizure activity or neurotoxicity. For example, when methamphetamine and $10 \mathrm{mg} / \mathrm{kg}$ of (-)chloroephedrine were co-administered only an increase in HR was observed. However, administration of the same dose of $(-)$-chloroephedrine alone produced a large bradycardia (200 bpm). Why the bradycardic response was blocked by the addition of methamphetamine is unclear.

The cardiovascular effects observed after administration of chloroephedrine may reflect the action of the chloroephedrine or the aziridine intermediate depending on the rate of cyclization to the aziridine. Currently, the rate and extent to which this occurs for $(+)$ - and $(-)$-chloroephedrine under neutral or physiological conditions is unknown. We have found the chloroephedrines to be stable for up to $24 \mathrm{~h}$ in aqueous solutions (Lekskulchai et al., in press). The stability of these compounds after oral administration or during pyrolysis remains to be determined. In general, the principal biological effects of betachloroamines and nitrogen mustards are cytotoxicity and irreversible binding to cell receptors. Through alkylation via the aziridine, the beta-chloroamines have been developed into clinically useful drugs (eg. phenoxybenzamine, melphalan) (Henkel et al., 1976) and investigational compounds such as mustard analogs of acetylcholine (Robinson, et al., 1975), or oxotremorine (Ringdahl et al., 1984) that have been used to investigate drug-receptor interactions.

These studies have shown that two possible contaminants of illicit methamphetamine manufacture, $(+)$ and $(-)$ chloroephedrine, have sympathomimetic properties and elicit a pattern of MAP and HR responses in conscious rats that is similar to that elicited by $(-)$-ephedrine. In addition, larger doses of these two compounds produce vagal activation. While the biological activity of these compounds in humans has not been evaluated, their potential danger should be investigated further. 


\section{Acknowledgements}

We would like to thank Ms Lisa Badon for her excellent technical support. This work was supported by a grant (DA 08255) from the National Institute on Drug Abuse.

\section{References}

Abrahams, T.P., Cuntapay, M.C., Varner, K.J., 1996. Sympathetic nerve responses elicited by cocaine in anesthetized and conscious rats. Physiol. Behav. 59, 109-115.

Allen, T.S., Kiser, W.O., 1987. Methamphetamine from ephedrine: chloroephedrines and aziridines. J. For. Sci. 32, 953-962.

Cantrell, T.S., John, B., Johnson, L., Allen, A.C., 1988. A study of impurities found in methamphetamine synthesized from ephedrine. For. Sci. Int. 39, 39-53.

Carvey, R.H., Reed, D., 1970. Intravenous amphetamine poisoning: report of three cases. J. For. Sci. Soc. 10, 109-112.

Dimson, S.B., 1934. The pressor actions of ephedrine and pseudoephedrine in man. In: Hampshire, C.H. (Ed.), Year-Book of Pharmacy. Pharmaceutical Press, London, pp. 23-31.

Drew, C.D.M., Knight, G.T., Hughs, D.T.D., Bush, M., 1978. Comparison of the effects of d-(-)-ephedrine and 1-(+)-pseudoephedrine on the cardiovascular and respiratory systems in man. Br. J. Clin. Pharm. 6, 221-225.

Fester, Uncle, 1999. Secrets of Methamphetamine Manufacture including Recipes for MDA, Ecstasy, and other Psychedelic Amphetamines, 5th edn. Loompanics Unlimited, Port Townsend, WA, pp. 114-118.

Fristrom, S., Marvola, M., Klinge, E., Halmekoski, J., 1997. Effect of acetyl derivatives of some sympathomimetic amines on the blood pressure of the rat. Acta Pharm. Tox. 40, 247-258.

Fruton, J.S., 1950. In: Elderfield, R.C. (Ed.), Ethylenimine in Heterocyclic Compounds. John Wiley and Sons, New York, pp. 61-67.

Fukunaga, T., Mizoi, Y., Adachi, J., Tatsuno, Y., 1987. Methamphetamine concentrations in blood, urine and organs of fatal cases after abuse. Jap. J. Legal Med. 41, 328-334.

Henkel, J.G., Portoghese, P.S., Miller, J.W., Lewis, P., 1976. Synthesis and adrenoreceptor blocking action of aziridinium ions derived from phenoxybenzamine and dibenamine. J. Med. Chem. 19, $6-10$.

Josefson, D., 1996. Herbal stimulant causes US deaths. Br. Med. J. 312, 1378-1379.

Karch, S.B., Stephens, B.G., Ho, C.-H., 1999. Methamphetamine-related deaths in San Francisco: demographic, pathologic and toxicologic profiles. J. For. Sci. 44, 359-368.
Kitagawa, H., Saito, H., 1975. Differences in the effect of ephedrine isomers on blood pressure in rats. Jpn. J. Pharm. 25, 473-475.

Kojima, T., Une, I., Yashiki, J., 1983. CI-mass fragmentographic analysis of methamphetamine and amphetamine in human autopsy tissues after acute methamphetamine poisoning. For. Sci. Int. 21, 253-258.

Konuma, K., 1994. Use and abuse of amphetamines in Japan. In: Cho, A.K., Segal, D.S. (Eds.), Amphetamine and its analogs. Academic Press, San Diego, pp. 423-424.

Lekskulchai, V., Carter, K., Poklis, A., Soine, W.H. GC/MS analysis of methamphetamine impurities: reactivity of $(+)$ - or $(-)$ chloroephedrine and cis- or trans-1,2-dimethyl-3-phenyl aziridine. J. Anal. Tox. (in press).

Maritn, W.R., Sloan, J.W., Sapira, J.D., Jasinski, D.R., 1970. Physiologic, subjective, and behavioral effects of amphetamine, methamphetamine, ephedrine, phenmetrazine, and methylphenidate in man. Clin. Pharm. Ther. 12, 245-258.

Matoba, R., Onishi, S., Shikata, I., 1985. Cardiac lesions in cases of sudden death in methamphetamine abusers. Heart Vessels 1, $298-300$.

Moore, K.A., Daniel, J.S., Fierro, M., Mozayani, A., Poklis, A., 1996. The detection of a metabolite of (-benzyl-Nmethylphenethylamine synthesis in a mixed drug fatality involving methamphetamine. J. For. Sci. 41, 524-526.

Noggle, F.T., DeRuiter, J., Clark, C.R., 1986. Liquid chromatographic determination of the enantiomeric composition of methamphetamine prepared from ephedrine and pseudoephedrine. Anal. Chem. 58, 1643-1648.

Pentel, P., 1984. Toxicity of over-the-counter stimulants. J. Am. Med. Assoc. 252, 1898-1903.

Ringdahl, B., Resul, B., Ehlert, F.J., Jenden, D.J., Dahlbom, R., 1984. The conversion of 2-chloroalkylamine analogues of oxotremorine to aziridinium ions and their interactions with muscarinic receptors in the guinea pig ileum. Mol. Pharm. 26, $170-179$.

Robinson, D.A., Taylor, J.G., Young, J.M., 1975. The irreversible binding of acetylcholine mustard to muscarinic receptors in intestinal smooth muscle of the guinea pig. Br. J. Pharm. 53, 363-370.

Skinner, H.F., 1990. Methamphetamine synthesis via hydriodic acid/ red phosphorous reduction of ephedrine. For. Sci. Int. 48, 123134.

Somers, V.K., Mark, A.L., 1996. The Bezold-Jarisch reflex. In: Robertson, D., Low, P.A., Ploinsly, R.J. (Eds.), Primer on the Autonomic Nervous System. Academic Press, San Diego, pp. 66-69.

Theoharides, T.C., 1997. Sudden death of a healthy college student related to ephedrine toxicity from a ma huang-containing drink. J. Clin. Psychopharm. 17, 437-439.

Thoren, P., 1979. Role of cardiac vagal c-fibers in cardiovascular control. Rev. Physiol. Biochem. Pharm. 86, 2-94.

Zahn, K.A., Li, R.L., Purssell, R.A., 1999. Cardiovascular toxicity after ingestion of "herbal ecstasy". J. Emerg. Med. 17, 289-291. 